\title{
Suppression of magnetostructural transition on GdSiGe thin film after thermal cyclings
}

\author{
A. L. Pires ${ }^{1}$, J. H. Belo ${ }^{1}$, I. T. Gomes ${ }^{1}$, R. L. Hadimani ${ }^{2,3}$, D. L. Schlagel ${ }^{3}$, T. A. \\ Lograsso $^{3,4}$, D. C. Jiles ${ }^{2,3}$, A. M. L. Lopes ${ }^{1}$, J. P. Araújo ${ }^{1 *}$, A. M. Pereira ${ }^{1 *}$ \\ ${ }^{1}$ IFIMUP and IN - Institute of Nanoscience and Nanotechnology, Departamento de Física e Astronomia da \\ Faculdade de Ciências da Universidade do Porto, Rua do Campo Alegre, 687, 4169-007 Porto, Portugal. \\ ${ }^{2}$ Department of Electrical and Computer Engineering, Iowa State University, Ames, Iowa 50011, USA \\ ${ }^{3}$ Ames Laboratory, US Department of Energy, Iowa State University, Ames, Iowa 50011, USA \\ ${ }^{4}$ Division of Materials Science and Engineering, Ames Laboratory, Ames, Iowa 50011, USA
}

The influence of thermal cycling on the microstructure, magnetic phase transition and magnetic entropy change of a $\mathrm{Gd}_{5} \mathrm{Si}_{1.3} \mathrm{Ge}_{2.7}$ thin film up to 1000 cycles is investigated. The authors found that after 1000 cycles a strong reduction of the crystallographic phase responsible for the magnetostructural transition (Orthorhombic II phase) occurs. This is attributed to chemical disorder, caused by the large number of expansion/compression cycles that the Orthorhombic II phase undergoes across the magnetostructural transition. The suppression of the magnetostructural transition corresponds to a drastic decrease of the thin film magnetic entropy change. These results reveal the importance of studying the thermal/magnetic cycles influence on magnetostructural transitions as they can damage a real-life device.

Keywords: Thermal Cycling, Magnetocaloric Effect, Thin Films, Microstructure

\section{Introduction}

In the last decade there has been an increasing demand towards clean energy and energy efficient devices, which will replace the more polluting and less efficient ones, such as refrigeration systems running on gases that contribute to the greenhouse effect [1]. In this framework, magnetic refrigeration systems emerge as the most prominent candidates to this technology, since they are more efficient, noiseless and are not considered harmful to the environment [2,3]. The magnetic refrigeration at room temperature is based on the magnetocaloric effect (MCE), which occurs in magnetic materials that exhibit

\footnotetext{
* Corresponding authors:

Dr. André Pereira, Tel.: +351 220402369; fax: +351220402406. E-mail address: ampereira@fc.up.pt; and

Dr. João Pedro Araújo, Tel.: +351-22 0402 362. E-mail address: jearaujo@fc.up.pt.
} 
a large magnetization change under the application of a magnetic field. They transform from a magnetically disordered state to an ordered state, and consequently undergo large magnetic entropy changes. In recent years, techniques such as doping and thermal treatments have been used to enhance the magnetocaloric effect [4-10]. It is consensual that the magnetic materials presenting first-order phase transitions are the ones exhibiting larger MCE, which is a key-parameter for their application in refrigeration systems. The $\mathrm{Gd}_{5}\left(\mathrm{Si}_{\mathrm{x}} \mathrm{Ge}_{1-\mathrm{x}}\right)_{4}$ [11-13], $\mathrm{La}\left(\mathrm{Fe}_{\mathrm{x}} \mathrm{Si}_{1-\mathrm{x}}\right)_{13}$ [14-17], $\mathrm{MnP}_{1-\mathrm{x}} \mathrm{As}_{\mathrm{x}}$, compounds belong to the group of first-order magnetic phase transition materials that exhibit a giant magnetocaloric effect (GMCE) $[4,6,18]$. However one drawback of these compounds is that their physical properties can change when subjected to magnetic/thermal cycling $[15,16,19]$. One of these changes is related to their MCE and in particular its magnitude during the cycling process, which is crucial for the magnetic refrigerator's performance. In magnetic refrigeration many parameters can influence the performance of these systems over time. For instance, for $\mathrm{LaFe}_{11.6} \mathrm{Si}_{1.4}$ it has been shown that an increase of the magnetic field promotes a reduction of thermal hysteresis upon increasing temperature [15]. The authors associated this result to the presence of a first-order magnetic transition that includes metastable states in addition to thermal equilibrium states. Also, properties such as electrical resistance [20-23], thermopower [24] and specific heat [25] have been reported as dependent of thermal cycling on $\mathrm{Gd}_{5}\left(\mathrm{Si}_{\mathrm{x}} \mathrm{Ge}_{1-\mathrm{x}}\right)_{4}$ alloys. Phenomena as disordered atomic structure and aging also have been associated to the reduction of both transition temperature and magnetic entropy change [26]. However, all studies performed on these compounds have been focused in the bulk form or in micrometric scale samples. Nowadays, driven by the prospect of producing miniaturized devices, from micro-refrigerators to magnetic/pressure/temperature sensors [2730], the interest on the study of MCE in materials with reduced dimensions has been growing considerably. Additionally, magnetocaloric thin films will be readily adapted to integrate micromagnetocaloric processes inside micro-electronic circuits [31], because of their advantageous higher surface to volume ratio. It has been found that ball milling technique allows to obtain micro/nanometer size particles which show a decrease of the overall magnetization values. This phenomenon can be due both to a magnetic moment blocking mechanism and an amorphization of the particles structure [32,33]. Recently, Zhang et al. [34] showed that the temperature and applied magnetic field cycles change the hysteresis of the MnAs nanocrystals. Therefore, it is important to understand the behavior of magnetocaloric materials at the nanoscale when they undergo a large number of thermal cycles [25]. In 
this way, the aim of presented work is to study the effect of consecutive thermal cycles on the microstructure, magnetization and magnetic entropy changes in $\mathrm{Gd}_{5} \mathrm{Si}_{1.3} \mathrm{Ge}_{2.7}$ thin film.

\section{Experimental methods}

The preparation details of the $\mathrm{Gd}_{5} \mathrm{Si}_{1.3} \mathrm{Ge}_{2.7}$ thin film on a $\mathrm{SiO}_{2}$-covered Si substrate using femtosecond pulsed laser ablation were described in Ref. [28]. The thermal cycling was performed by immersing the $\mathrm{Gd}_{5} \mathrm{Si}_{1.3} \mathrm{Ge}_{2.7}$ thin film (thickness $763 \pm 25 \mathrm{~nm}$ ) in a liquid nitrogen bath. In each cycle, the film was immersed for 60 seconds in order to ensure thermal equilibrium with the bath and afterwards it was removed immediately. This cycle was repeated 1000 times and between consecutive dips the sample rested at room temperature during 60 seconds. Such procedure was repeated for two samples: Sample 1 and Sample 2. Scanning Electron Microscopy, SEM (Schottky - FEG-ESEM/EDS/EBSD FEI Quanta 400 FEG ESEM/EDAX Genesis X4M - with $15.00 \mathrm{kV}$ in secondary electrons mode) was used to evaluate the microstructure of the thin films before cycling and after 50 and 450 thermal cycles. X-ray diffraction patterns, XRD, were also performed before and after 450 and 1000 cycles in a MiniFlex600 Rigaku Diffractometer with a Bragg-Brentano theta-2 theta configuration (condition: current of $15 \mathrm{~mA}$, voltage of $40 \mathrm{kV}$ and $\mathrm{K} \alpha$ radiation with a wavelength of $\lambda=1.54056 \AA$ ). The magnetic measurements was performed in a commercial (Quantum Design MPMS-5S) Superconducting Quantum Interference Device magnetometer. The magnetic measurements performed included isothermal, moment vs field, i.e. $\mathrm{M}(\mathrm{H})$ curves up to $5 \mathrm{~T}$. The magnetic entropy change curves $[-\Delta \operatorname{Sm}(\mathrm{T})]$ were estimated through the application of the Maxwell relation [35]. Temperature dependence of magnetization $M(T)$ was measured before and after 450 and 1000 cycles. Sample 2 was inspected in order to confirm sample 1 results.

\section{Results and Discussion}

Figure 1 shows the SEM of the thin film top view, obtained for the same specific region before cycling and after 50 and 450 cycles. From Figures $1 \mathrm{a} 1)$ - b1), it is observed that the micro-morphology of the thin film presents a clustering of spherical particles that remains almost unaltered during the thermal cycling for the sample after 50 cycles. After 450 cycles (Figure $1 \mathrm{c} 1$ ) the morphology remains the same but is not possible to identify the same initial spot, i.e. the thermal cycling induced movement at the surface of the thin films. We attribute such small changes to the induced strain promoted by the thermal cycles that through the nanoparticles expansion/shrinking can promote their slight movement along the surface. When performing the size histogram and using a Log-Normal distribution, similar mean sizes of 
$108 \mathrm{~nm}$ before cycling, and $112 \mathrm{~nm}$ after 50 and 450 cycles, within the error, are obtained, Figure 1a2) c2). The major change is on the full width at half maximum of the distribution that decreases with the cycling, showing a narrower size distribution.

The thin film $\mathrm{M}(\mathrm{T})$ behavior, before and after thermal cycles, is shown in Figure 2a). It can be observed that the $\mathrm{Gd}_{5} \mathrm{Si}_{1.3} \mathrm{Ge}_{2.7}$ thin film exhibits two magnetic transitions [9,28,36]. At $\mathrm{T} \sim 249 \mathrm{~K}$ a magnetic transition occurs, corresponding to a pure paramagnetic to ferromagnetic second-order transition (orthorhombic $\mathrm{O}(\mathrm{I})$ crystal structure is retained). At lower temperature, $\mathrm{T} \sim 197 \mathrm{~K}$, a structural and a magnetic transition occur simultaneously - magnetostructural transition (MST) - from a paramagnetic orthorhombic $\mathrm{O}(\mathrm{II})$ to a ferromagnetic $\mathrm{O}(\mathrm{I})$ phase, as previously reported by the authors [37]. Furthermore, in this first-order transition, thermal hysteresis is observed, where the transition temperatures for heating and cooling are $\sim 197 \mathrm{~K}$ and $\sim 187 \mathrm{~K}$, respectively $[6,36]$. The authors remark that this stoichiometry, $\mathrm{Gd}_{5} \mathrm{Si}_{1.3} \mathrm{Ge}_{2.7}$, presents one of the highest volume changes throughout the structural transition from $[\mathrm{O}(\mathrm{II}), \mathrm{PM}] \rightarrow[\mathrm{O}(\mathrm{I}), \mathrm{FM}]$ leading also to one of the highest magnetic entropy variation.

After 450 cycles, the $\mathrm{M}(\mathrm{T})$ exhibit a similar behavior as the as-deposited thin film, (Figure 2a)) overlapping in the [210-300] $\mathrm{K}$ temperature range. The thermal cycling does not seem to affect the second-order transition that occurs at $249 \mathrm{~K}$. The major difference arises at lower temperatures $(\sim 197 \mathrm{~K}$, on heating) because thermal cycling causes a decrease in the amount of phase that undergoes the MST. This statement is confirmed by the $\mathrm{M}(\mathrm{H})$ loops obtained at the same temperature leading to a $21 \%$ decrease of the magnetic hysteresis after 450 cycles (Figure 3 a) and b)). Such decrease is another indication that thermal cycling is mainly actuating on the MST. When increasing the number of thermal cycles to 1000 , the second-order phase transition at $249 \mathrm{~K}$ remains unaltered. However, it is possible to observe the complete suppression of the MST, at lower temperatures. Such suppression can be attributed to chemical disorder promoted by the internal strain and stress cycles that the atoms are subjected to along the thermal cycles. Also the $\mathrm{M}(\mathrm{H})$ curves at $5 \mathrm{~K}$ (Figure $3 \mathrm{c})$ ), clearly change from a typical ferromagnet (before cycling) towards a paramagnetic behavior (already seen in the 450 cycles curve and more accentuated in the 1000 cycles curve).

In order to study the atomic structure, XRD analysis was carried out at room temperature (Figure $2 b$ ). The XRD patterns show peaks corresponding to the $\mathrm{O}(\mathrm{I})$ phase $\left(\mathrm{Gd}_{5} \mathrm{Si}_{4}\right)$ [38] and $\mathrm{O}(\mathrm{II})$ phase $\left(\mathrm{Gd}_{5} \mathrm{Ge}_{4}\right)$ [39] identified with “*” and "+”, respectively. Upon comparison, it is observed that there is an overall peak-intensity decrease after cycling. However such decrease is more drastic for the O(II) phase peaks, 
such as ( $\left.\begin{array}{lll}3 & 1 & 1\end{array}\right)$ and $\left(\begin{array}{lll}1 & 6 & 4\end{array}\right)$, as their intensities are clearly reduced after 450 cycles and are absent after 1000 cycles. This corroborates the hypothesis of $\mathrm{O}(\mathrm{II})$ phase loss, as suggested by the magnetization results. At lower angles a shift of the peaks is also observed which can be related with induced strain after cycling. In particular, the XRD pattern for the sample after 1000 cycles reveals the absence of the O(II) phase peaks. Regarding the $\mathrm{O}(\mathrm{I})$ phase peaks, only one remains, indexed with the $\left(\begin{array}{lll}2 & 1 & 1\end{array}\right)$ reflection.

The saturation moments $\left(\mu_{\mathrm{S}}\right)$ were calculated from the magnetization curves at $5 \mathrm{~K}$ (Figure $3 \mathrm{c}$ )) considering the $\mathrm{Gd}_{5} \mathrm{Si}_{1.3} \mathrm{Ge}_{2.7}$ bulk density $\left(7700 \mathrm{Kg} / \mathrm{m}^{3}\right)$ [6]. The estimated values for the sample before and after 450 and 1000 cycles are $6.7 \pm 0.5,6.2 \pm 0.5$ and $6.0 \pm 0.5 \mu_{\mathrm{B}}$, respectively. These values are close to the theoretical $7 \mu_{\mathrm{B}}$ saturation magnetic moment of pure $\mathrm{Gd}^{3+}$ ions [6]. This result excludes clearly the possibility of substantial mass loss during the cycling process.

The magnetic entropy variation was estimated by integrating the Maxwell relation on the $\mathrm{M}(\mathrm{H})$ curves for before and after 50, 200, 250, 450 and 1000 cycles, as is shown in Figure 4a) in the [170, 225] K temperature range, for sample 1 and sample 2. For all curves the peak value of the magnetic entropy change, $-\Delta \mathrm{S}_{\mathrm{m}}{ }^{\max }$, occurs around the same temperature, $\mathrm{T} \sim 192.5 \mathrm{~K}$, as a consequence of the MST occurring systematically at this temperature [28].

Concerning the dependence of $\Delta \mathrm{S}_{\mathrm{m}}$ magnitude as a function of the number of cycles, before cycling the sample 1 has a maximum magnetic entropy change $-\Delta \mathrm{S}_{\mathrm{m}}{ }^{\max } \sim 64 \mathrm{mJK}^{-1} \mathrm{~cm}^{-3}$, then decreases after 50, 200 and 250 cycles by $10 \%\left(58 \mathrm{mJK}^{-1} \mathrm{~cm}^{-3}\right), 15 \%\left(55 \mathrm{mJK}^{-1} \mathrm{~cm}^{-3}\right)$ and $17 \%\left(53 \mathrm{mJK}^{-1} \mathrm{~cm}^{-3}\right)$ respectively, decreasing at then, 450 cycles, by $19 \%$ of the $-\Delta \mathrm{S}_{\mathrm{m}}{ }^{\max }$ value $\left(\sim 52 \mathrm{mJK}^{-1} \mathrm{~cm}^{-1}\right)$. After 1000 cycles the magnetic entropy change reduces drastically to $\sim 12 \mathrm{mJK}^{-1} \mathrm{~cm}^{-1}$ (corresponding to a reduction of $81 \%$ ). In order to check this apparent linear behavior the same procedure was applied to sample 2. The major difference is the 450 cycles point, which shows a more accentuated decrease of $37 \%\left(40.48 \mathrm{mJK}^{-1} \mathrm{~cm}^{-1}\right)$. Whereas, the 1000 cycles point is in accordance with the sample 1 point $\left(\sim 12.7 \mathrm{mJK}^{-1} \mathrm{~cm}^{-1}\right)$. Hence, sample 2 behavior confirms sample 1 linear decrease of the $\Delta \mathrm{S}_{\mathrm{m}}{ }^{\mathrm{max}}$ values with the number of cycles, with an $-0.05 \mathrm{mJK}^{-1} \mathrm{~cm}^{-1} /$ cycle slope, reaching the $\Delta \mathrm{S}_{\mathrm{m}}{ }^{\max }$ base value of $\sim 12 \mathrm{mJK}^{-1} \mathrm{~cm}^{-1}$ at the end of the 1000 cycles.

Concerning the refrigerant capacity, $\mathrm{RCP}_{\mathrm{FWHM}}$, we observed a decrease with the number of cycles, with a maximum variation for 450 cycles $\left(\mathrm{RCP}_{\mathrm{FWHM}}\right.$ Before Cycling $=1239 \mathrm{mJcm}^{-3}$; $\mathrm{RCP}_{\mathrm{FWHM}}$ After 450 
Cycling $=1127 \mathrm{mJcm}^{-3}$ ). After 1000 cycles an estimation of $\mathrm{RCP}_{\mathrm{FWHM}}$ was no longer possible due to the large FWHM.

Analysing the obtained results it outcomes that thermal cycling is playing an important role on driving the structural transition. This is evident on the $M(T)$ curves - (Figure 2a), due to the systematic decrease in the hysteresis area, until its complete disappearance after 1000 cycles for both samples. Regarding the evolution of the the $-\Delta \mathrm{S}_{\mathrm{m}}{ }^{\text {max }}$ values, a linear decrease was obtained with number of cycles (Figure 4b).

In these materials, the structural transition induces an abrupt volume change $(\sim 1 \%)$, where the smallest volume phase stabilizes at lower temperatures, i.e., $[\mathrm{V}(\mathrm{O}(\mathrm{I}))<\mathrm{V}(\mathrm{O}(\mathrm{II}))][26]$. During the cooling process the appearance of free space due to the contraction of the unit cells is expected, thus releasing internal strain that can be formed during the thin film growth. For the reverse process, i.e. during heating, a significant increase in the unit cell volume will lead to a stress increase arising from the volume cell expansion, thus originating the arrest of the structural transition $[23,28]$. In fact, this mechanism has been referenced several times for bulk materials to explain changes in their properties, namely morphological, magnetic and transport [23]. Also the same explanation was used for other family of systems showing drastic volume changes [28]. In the $\mathrm{R}_{5}(\mathrm{Si}, \mathrm{Ge})_{4}$ bulk compounds family, we highlight the works from Casanova and co-authors and Perez and co-authors which demonstrated that the metastability, responsible for the thermal hysteresis, is reduced when the number of thermal cycles increases $[25,40]$. Furthermore, a previous study on the $\mathrm{Gd}_{5}\left(\mathrm{Si}_{0.1} \mathrm{Ge}_{0.9}\right)_{4}$ electrical resistivity behavior has also shown a thermal hysteresis area reduction as the number of cycles increase: the number of defects, the atomic chemical disorder, domain reorientation or the preferential $\mathrm{Si} / \mathrm{Ge}$ sites were assigned as the major causes of the metastability reduction $[22,23]$. The local behavior of the magnetic relaxation (across the field increase and decrease) of the $\mathrm{Gd}_{5} \mathrm{Ge}_{4}$ first-order transition has also been investigated [41]. It was concluded that the transition at lower temperatures is arrested and a metastable equilibrium (disorder-influenced first-order transition) is obtained, leading to a significant decrease in magnetic relaxation. On the other hand, at high temperatures, only a slight decrease was observed due to a faster process towards the metastable equilibrium. At these temperatures the activation over the excitation strain energy barriers is facilitated [41-43]. In fact, the system attains the equilibrium in a faster and more homogeneous way, i.e., tends to reduce the dissipated energy and therefore to reduce the hysteresis [40]. On other systems exhibiting a MST, such as in $\mathrm{Ni}_{54.3} \mathrm{Mn}_{20.1} \mathrm{Ga}_{25.6}$ alloys [19], a decrease of the magnetic moment with the number of 
cycles was also detected and associated with an arrest of the small volume phase (the austenite phase in that case), caused by local strain. This phenomenon is also extended to $\mathrm{La}(\mathrm{Fe}, \mathrm{Si})_{13}$ family of compounds where a decrease of the MCE in a few cycles was observed in the bulk form [30]. In another study, the stress relaxation, accompanied by a short-range order structural change, is responsible for the decrease of the $\Delta \mathrm{Sm}[26]$.

Therefore, we believe that in the present case the stress/strain generated during the cycling, across first-order phase transition is responsible for the decrease of the entropy as a function of thermal cycles. The decrease of the particle size can also be responsible for this reduction [32]. Hence with thermal cycles the $\mathrm{O}$ (II) phase is reduced due to the intense build up stress that the sample is subjected. Furthermore a phenomenon such as structural disorder might also be playing a role on the loss of the O(II) phase (confirmed by magnetic and structural data) and consequently the absence of the magnetostructural transition. This loss is accompanied with an increase of paramagnetism in the samples, as can be observed in Figure 3a) and b). In fact, the $\mathrm{M}(\mathrm{H})$ curve at $5 \mathrm{~K}$ after 1000 cycles shows a distinct behavior, corresponding to a clear decrease of ferromagnetism. The XRD data corroborate this assumption as nearly reflections disappear after 1000 cycles. Recently Zhang et al. [34], performed cooling cycles on MnAs nanocrystals (down to liquid nitrogen temperature), and have also reported a loss of hysteresis and loss of MST promoted by temperature and field cycles. It is important to remark that, in comparison with the bulk form, where the crystallographic grain surface area is several micrometersquare, the nanoparticles surface can be up to three orders of magnitude lower, which enhances (by three orders of magnitude) the stress that these nanoparticles are subjected to along the magnetostructural transition. This behavior has a parallel with the ball milling process used for a top-down approach to produce $\mathrm{Gd}_{5}(\mathrm{Si}, \mathrm{Ge})_{4}$ nanoparticles, where disorder and amorphization are also associated with a decrease of magnetic ordering and consequent decrease of MCE. [26,32,44]. Also, Vishnoi et al. [44], reported the degradation of the first-order transition and the amorphization on the $\mathrm{Ni}_{50} \mathrm{Mn}_{35.6} \mathrm{Sn}_{14.4}$ films with the increase of ion irradiation, i.e, increasing stress.

\section{Conclusions}

In summary, in this work we report the effects of thermal cycling on $\mathrm{Gd}_{5} \mathrm{Si}_{1.3} \mathrm{Ge}_{2.7}$ thin film properties. The magnetization and structural investigation in this thin film showed a decrease/disappearance of the $\mathrm{O}(\mathrm{II})$ phase. A linear decrease of the $-\Delta \mathrm{S}_{\mathrm{m}}{ }^{\mathrm{max}}$ with the number of cycles was 
observed. After 1000 cycles a complete loss of the O(II) phase is observed, attributed to chemical disorder induced by the internal strain and stress during the thermal cycles. Therefore, thermal cycles promote the loss of crystallinity, concomitant with a decrease of the magnetization and the magnetic entropy change. It is clear the partial transformation of the ferromagnetic phase to a paramagnetic one. In the future, studies using acoustic emission may be useful to better understand these results, in particular for the investigation of the kinetics of the structural transition and the crystallographic phase evolution in such thin films. Also, the behavior of freestanding nanoparticles (not constrained in a substrate) needs to be studied. Finally, a comparison with a non-granular thin film would give also great insight for the full comprehension of the operating stress/strain mechanisms. Also the complete understanding of the effect of magnetostructural transition combined with the thermal cycling for different thicknesses needs to be studied.

\section{ACKNOWLEDGMENTS}

The authors acknowledge FCT for financial support through the projects: PTDC/CTMNAN/115125/2009, EXPL/EMS-ENE/2315/2013， FEDER/POCTIn0155/94， JF/00686/2014 and CERN/FIS-NUC/0004/2015. J. H. Belo thanks FCT for the Grant SFRH/BD/88440/2012. A. M. Pereira, I. T. Gomes and A. M. L. Lopes acknowledge the project NORTE-070124-FEDER-000070 for the financial support. A. L. Pires thanks for the Grant: PEst-OE/FIS/UI0275/2014 and Incentivo/FIS/UI0275/2014. Work at Ames Laboratory was supported by the U.S. Department of Energy,

Office of Basic Energy Sciences, Division of Materials Science and Engineering. Ames Laboratory is operated for DOE by Iowa State University under Contract No. DE-AC02-07CH11358.

\section{REFERENCE}

[1] E. Brück, Developments in magnetocaloric refrigeration, J. Phys. D. Appl. Phys. 38 (2005) R381-R391.

[2] K.A. Gschneidner, V.K. Pecharsky, Thirty years of near room temperature magnetic cooling: Where we are today and future prospects, Int. J. Refrig. 31 (2008) 945-961.

[3] J.G. Romero, R.F. Garcia, A.M. Catoira, M.R. Gómez, Magnetocaloric effect: A review of the thermodynamic cycles in magnetic refrigeration, Renew. Sustain. Energy Rev. 17 (2013) 74-82.

[4] Y. Mudryk, V.K. Pecharsky, K.A. Gschneidner, Including Actinides, Elsevier, 2014.

[5] A.C.S. Hamilton, G.I. Lampronti, S.E. Rowley, S.E. Dutton, Enhancement of the magnetocaloric effect driven by changes in the crystal structure of Al-doped GGG, $\mathrm{Gd}_{3} \mathrm{Ga}_{5-\mathrm{x}} \mathrm{Al}_{\mathrm{x}} \mathrm{O}_{12}(0 \leq \mathrm{x} \leq 5)$., $\mathrm{J}$. 
Phys. Condens. Matter. 26 (2014) 116001.

[6] K.A. Gschneidner, V.K. Pecharsky, A.O. Tsokol, Recent developments in magnetocaloric materials, Reports Prog. Phys. 68 (2005) 1479-1539.

[7] J.Q. Li, W.A. Sun, Y.X. Jian, Y.H. Zhuang, W.D. Huang, J.K. Liang, The giant magnetocaloric effect of $\mathrm{Gd}_{5} \mathrm{Si}_{1.95} \mathrm{Ge}_{2.05}$ enhanced by Sn doping, J. Appl. Phys. 100 (2006) 073904.

[8] A.M. Pereira, A.M. dos Santos, C. Magen, J.B. Sousa, P.A. Algarabel, Y. Ren, C. Ritter, L. Morellon, M.R. Ibarra, Understanding the role played by $\mathrm{Fe}$ on the tuning of magnetocaloric effect in $\mathrm{Tb}_{5} \mathrm{Si}_{2} \mathrm{Ge}_{2}$, Appl. Phys. Lett. 98 (2011) 122501.

[9] J.H. Belo, A.M. Pereira, J. Ventura, G.N.P. Oliveira, J.P. Araújo, P.B. Tavares, L. Fernandes, P.A. Algarabel, C. Magen, L. Morellon, M.R. Ibarra, Phase control studies in $\mathrm{Gd}_{5} \mathrm{Si}_{2} \mathrm{Ge}_{2}$ giant magnetocaloric compound, J. Alloys Compd. 529 (2012) 89-95.

[10] J.H. Belo, A.M. Pereira, J.P. Araújo, C. de la Cruz, A.M. dos Santos, J.N. Gonçalves, V.S. Amaral, L. Morellon, M.R. Ibarra, P.A. Algarabel, C. Magen, Tailoring the magnetism of $\mathrm{Tb}_{5} \mathrm{Si}_{2} \mathrm{Ge}_{2}$ compounds by La substitution, Phys. Rev. B. 86 (2012) 014403.

[11] M. Manivel Raja, R. Gopalan, D.M. Rajkumar, R. Balamuralikrishnan, V. Chandrasekaran, K.G. Suresh, K. Hono, Phase relationship, microstructure and magnetocaloric effect in $\mathrm{Gd}_{1-\mathrm{x}}\left(\mathrm{Si}_{0.5} \mathrm{Ge}_{0.5}\right)_{\mathrm{x}}$ alloys, J. Phys. D. Appl. Phys. 41 (2008) 055008.

[12] A. Pires, J. Belo, A. Lopes, I. Gomes, L. Morellón, C. Magen, P.A. Algarabel, M.R. Ibarra, A.M. Pereira, J.P. Araújo, Phase Competitions behind the Giant Magnetic Entropy Variation: $\mathrm{Gd}_{5} \mathrm{Si}_{2} \mathrm{Ge}_{2}$ and $\mathrm{Tb}_{5} \mathrm{Si}_{2} \mathrm{Ge}_{2}$ Case Studies, Entropy. 16 (2014) 3813-3831.

[13] V.K. Pecharsky, K.A. Gschneidner, Giant Magnetocaloric Effect in $\mathrm{Gd}_{5}\left(\mathrm{Si}_{2} \mathrm{Ge}_{2}\right)$, Phys. Rev. Lett. 78 (1997) 4494-4497.

[14] K. Morrison, J. Lyubina, J.D. Moore, A.D. Caplin, K.G. Sandeman, O. Gutfleisch, L.F. Cohen, Contributions to the entropy change in melt-spun $\mathrm{LaFe}_{11.6} \mathrm{Si}_{1.4}$, J. Phys. D. Appl. Phys. 43 (2010) 132001.

[15] K.P. Skokov, K.-H. Müller, J.D. Moore, J. Liu, A.Y. Karpenkov, M. Krautz, O. Gutfleisch, Influence of thermal hysteresis and field cycling on the magnetocaloric effect in $\mathrm{LaFe}_{11.6} \mathrm{Si}_{1.4}, \mathrm{~J}$. Alloys Compd. 552 (2013) 310-317.

[16] A. Waske, L. Giebeler, B. Weise, A. Funk, M. Hinterstein, M. Herklotz, K. Skokov, S. Fahler, O. Gutfleisch, J. Eckert, Asymmetric first-order transition and interlocked particle state in magnetocaloric La(Fe,Si) ${ }_{13}$, Phys. Status Solidi - Rapid Res. Lett. 9 (2015) 136-140.

[17] E. Lovell, A.M. Pereira, A.D. Caplin, J. Lyubina, L.F. Cohen, Dynamics of the First-Order Metamagnetic Transition in Magnetocaloric $\mathrm{La}(\mathrm{Fe}, \mathrm{Si})_{13}$ : Reducing Hysteresis, Adv. Energy Mater. 5 (2015) 1401639.

[18] J. Liu, T. Gottschall, K.P. Skokov, J.D. Moore, O. Gutfleisch, Giant magnetocaloric effect driven by structural transitions., Nat. Mater. 11 (2012) 620-6.

[19] R.A. Booth, S.A. Majetich, The magnetocaloric effect in thermally cycled polycrystalline Ni-MnGa, J. Appl. Phys. 111 (2012) 07A933.

[20] E. Levin, A. Pecharsky, V. Pecharsky, K. Gschneidner, Transformations in the $\operatorname{Gd}_{5}\left(\mathrm{Si}_{1.95} \mathrm{Ge}_{2.05}\right)$ alloy induced by the temperature and magnetic-field cycling through the first-order magneticmartensitic phase transition, Phys. Rev. B. 63 (2001) 064426.

[21] R.L. Hadimani, Y. Melikhov, J.E. Snyder, D.C. Jiles, Anomalous Behavior in Electrical Transport Properties in Single-Crystal Gd5Si1.8Ge2.2 and Polycrystalline $\mathrm{Gd}_{5} \mathrm{Si}_{2.09} \mathrm{Ge}_{1.91}$, IEEE 
Trans. Magn. 45 (2009) 4368-4371.

[22] J.B. Sousa, M.E. Braga, F.C. Correia, F. Carpinteiro, Anomalous behavior of the electrical resistivity in the giant magnetocaloric compound $\mathrm{Gd}_{5}\left(\mathrm{Si}_{0.1} \mathrm{Ge}_{0.9}\right)_{4}$, Phys. Rev. B. 67 (2003) 134416.

[23] J.B. Sousa, A.M. Pereira, F.C. Correia, J.M. Teixeira, J.P. Araújo, R.P. Pinto, M.E. Braga, L. Morellon, P.A. Algarabel, C. Magen, M.R. Ibarra, Multi-step and anomalous reproducible behaviour of the electrical resistivity near the first-order magnetostructural transition of $\mathrm{Gd}_{5}\left(\mathrm{Si}_{0.1} \mathrm{Ge}_{0.9}\right)_{4}$, J. Phys. Condens. Matter. 17 (2005) 2461-2476.

[24] J.B. Sousa, M.E. Braga, F.C. Correia, F. Carpinteiro, L. Morellon, P.A. Algarabel, R. Ibarra, Thermopower behavior in the $\mathrm{Gd}_{5}\left(\mathrm{Si}_{0.1} \mathrm{Ge}_{0.9}\right)_{4}$ magnetocaloric compound from 4 to $300 \mathrm{~K}, \mathrm{~J}$. Appl. Phys. 91 (2002) 4457.

[25] F. Casanova, A. Labarta, X. Batlle, F.J. Pérez-Reche, E. Vives, L. Mañosa, A. Planes, Direct observation of the magnetic-field-induced entropy change in $\mathrm{Gd}_{5}\left(\mathrm{Si}_{\mathrm{x}} \mathrm{Ge}_{1-\mathrm{x}}\right)_{4}$ giant magnetocaloric alloys, Appl. Phys. Lett. 86 (2005) 262504.

[26] Q. Luo, W.H. Wang, Magnetocaloric effect in rare earth-based bulk metallic glasses, J. Alloys Compd. 495 (2010) 209-216.

[27] M. Kumaresavanji, C.T. Sousa, A. Pires, A.M. Pereira, A.M.L. Lopes, J.P. Araujo, Room temperature magnetocaloric effect and refrigerant capacitance in $\mathrm{La}_{0.7} \mathrm{Sr}_{0.3} \mathrm{MnO}_{3}$ nanotube arrays, Appl. Phys. Lett. 105 (2014) 083110.

[28] R.L. Hadimani, J.H.B. Silva, A.M. Pereira, D.L. Schlagel, T.A. Lograsso, Y. Ren, X. Zhang, D.C. Jiles, J.P. Araújo, $\mathrm{Gd}_{5}(\mathrm{Si}, \mathrm{Ge})_{4}$ thin film displaying large magnetocaloric and strain effects due to magnetostructural transition, Appl. Phys. Lett. 106 (2015) 032402.

[29] M.F. Ozaydin, H. Liang, Multi-energy conversion of $\mathrm{Gd}_{5}\left(\mathrm{Si}_{2} \mathrm{Ge}_{2}\right)$-poly (vinylidene fluoride), a hybrid material, Appl. Phys. Lett. 105 (2014) 062903.

[30] J. Lyubina, R. Schäfer, N. Martin, L. Schultz, O. Gutfleisch, Novel design of La(Fe,Si) 13 alloys towards high magnetic refrigeration performance., Adv. Mater. 22 (2010) 3735-9.

[31] Q. Zhang, S. Thota, F. Guillou, P. Padhan, V. Hardy, A. Wahl, W. Prellier, Magnetocaloric effect and improved relative cooling power in $\mathrm{La}_{0.7} \mathrm{Sr}_{0.3} \mathrm{MnO}_{3} / \mathrm{SrRuO}_{3}$ superlattices., J. Phys. Condens. Matter. 23 (2011) 052201.

[32] G. Giovanna do Couto, V. Svitlyk, M. Jafelicci, Y. Mozharivskyj, Bulk and high-energy ballmilled $\mathrm{Gd}_{5} \mathrm{Si}_{2} \mathrm{Ge}_{2}$ : Comparative study of magnetic and magnetocaloric properties, Solid State Sci. 13 (2011) 209-215.

[33] A.L. Pires, J.H. Belo, J. Turcaud, G.N.P. Oliveira, J.P. Araújo, A. Berenov, L.F. Cohen, A.M.L. Lopes, A.M. Pereira, Influence of short time milling in $\mathrm{R}_{5}(\mathrm{Si}, \mathrm{Ge})_{4}, \mathrm{R}=\mathrm{Gd}$ and $\mathrm{Tb}$, magnetocaloric materials, Mater. Des. 85 (2015) 32-38.

[34] Y. Zhang, R. Regmi, Y. Liu, G. Lawes, S.L. Brock, Phase-coexistence and thermal hysteresis in samples comprising adventitiously doped MnAs nanocrystals: Programming of aggregate properties in magnetostructural nanomaterials, ACS Nano. 8 (2014) 6814-6821.

[35] L. Caron, Z.Q. Ou, T.T. Nguyen, D.T. Cam Thanh, O. Tegus, E. Brück, On the determination of the magnetic entropy change in materials with first-order transitions, J. Magn. Magn. Mater. 321 (2009) 3559-3566.

[36] A.O. Pecharsky, K.A. Gschneidner, V.K. Pecharsky, C.E. Schindler, The room temperature metastable/stable phase relationships in the pseudo-binary $\mathrm{Gd}_{5} \mathrm{Si}_{4}-\mathrm{Gd}_{5} \mathrm{Ge}_{4}$ system, J. Alloys Compd. 338 (2002) 126-135. 
[37] A.L. Pires, J.H. Belo, I.T. Gomes, R.L. Hadimani, D.C. Jiles, L. Fernandes, P.B. Tavares, J.P. Araújo, A.M.L. Lopes, A.M. Pereira, Annealing influence on the magnetostructural transition in $\mathrm{Gd}_{5} \mathrm{Si}_{1.3} \mathrm{Ge}_{2.7}$ thin films, Mater. Lett. 159 (2015) 301-304.

[38] J.E. Iglesias, H. Steinfink, The crystal structure of $\mathrm{Gd}_{5} \mathrm{Si}_{4}$, J. Less Common Met. 26 (1972) 4552.

[39] H.F. Yang, G.H. Rao, G.Y. Liu, Z.W. Ouyang, W.F. Liu, X.M. Feng, W.G. Chu, J.K. Liang, Crystal structures of compounds in the pseudobinary system $\mathrm{Gd}_{5} \mathrm{Ge}_{4}-\mathrm{La}_{5} \mathrm{Ge}_{4}$, J. Alloys Compd. 361 (2003) 113-117.

[40] F.-J. Pérez-Reche, F. Casanova, E. Vives, L. Mañosa, A. Planes, J. Marcos, X. Batlle, A. Labarta, Acoustic emission across the magnetostructural transition of the giant magnetocaloric $\mathrm{Gd}_{5} \mathrm{Si}_{2} \mathrm{Ge}_{2}$, Phys. Rev. B. 73 (2006) 014110.

[41] Z. Ouyang, V. Pecharsky, K. Gschneidner, D. Schlagel, T. Lograsso, Field step size and temperature effects on the character of the magnetostructural transformation in a $\mathrm{Gd}_{5} \mathrm{Ge}_{4}$ single crystal, Phys. Rev. B. 76 (2007) 134406.

[42] J.D. Moore, G.K. Perkins, K. Morrison, L. Ghivelder, M.K. Chattopadhyay, S.B. Roy, P. Chaddah, K.A. Gschneidner, V.K. Pecharsky, L.F. Cohen, Local probing of arrested kinetics in $\mathrm{Gd}_{5} \mathrm{Ge}_{4}$, J. Phys. Condens. Matter. 20 (2008) 465212.

[43] M. Chattopadhyay, M. Manekar, A. Pecharsky, V. Pecharsky, K. Gschneidner, J. Moore, G.K. Perkins, Y.V. Bugoslavsky, S.B. Roy, P. Chaddah, L.F. Cohen, Metastable magnetic response across the antiferromagnetic to ferromagnetic transition in $\mathrm{Gd}_{5} \mathrm{Ge}_{4}$, Phys. Rev. B. 70 (2004) 214421.

[44] R. Vishnoi, R. Singhal, K. Asokan, D. Kanjilal, D. Kaur, Ion irradiation induced modifications of nanostructured Ni-Mn-Sn ferromagnetic shape memory alloy thin films, Thin Solid Films. 520 (2011) 1631-1637. 


\section{Figure 1}
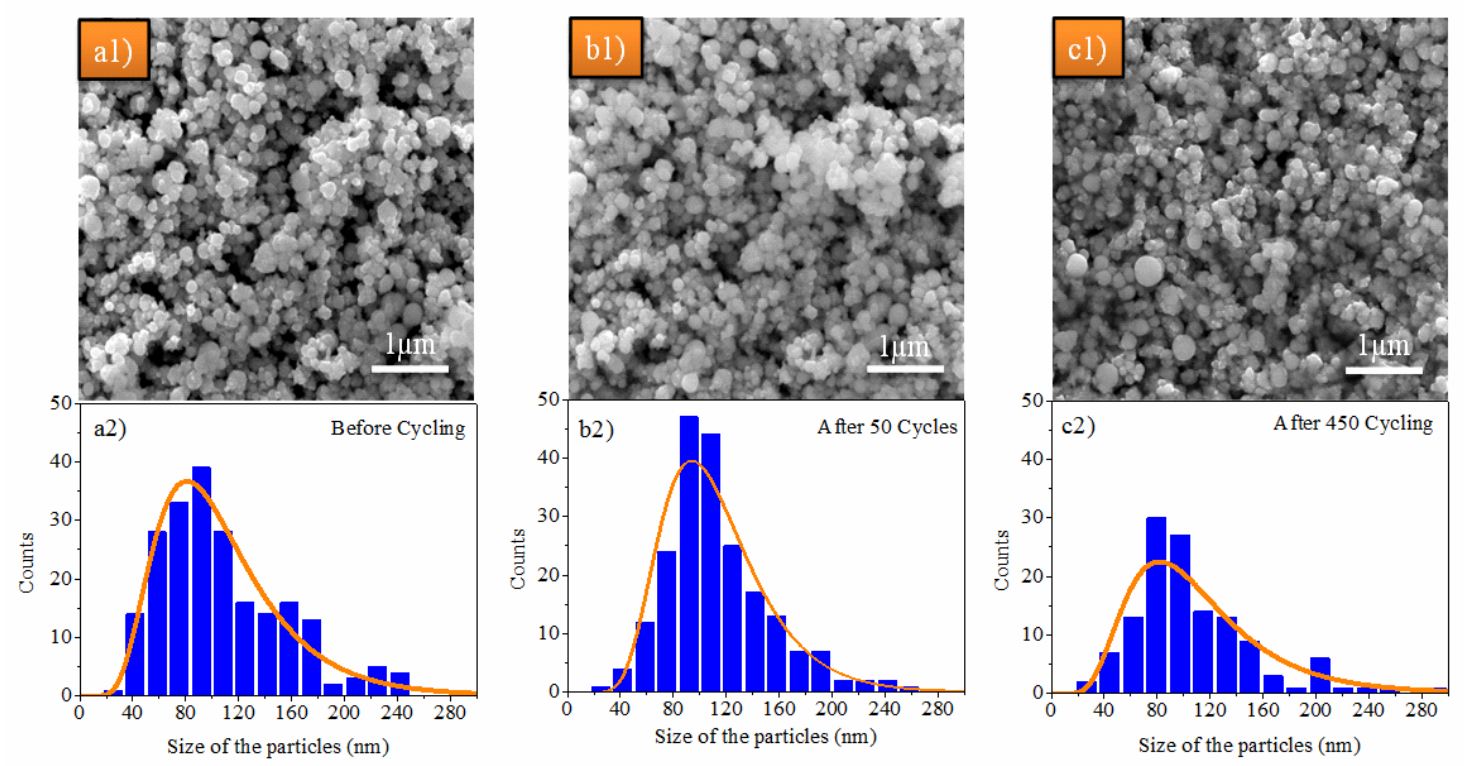

Figure 1 (color online): $\mathrm{Gd}_{5} \mathrm{Si}_{1.3} \mathrm{Ge}_{2.7}$ thin film SEM images: (a1) before thermal cycling; (b1) after 50 thermal cycles and (c1) after 450 thermal cycles; and the corresponding histograms (a2, b2 and c2) fitted with Log-Normal distributions. 


\section{Figure 2}
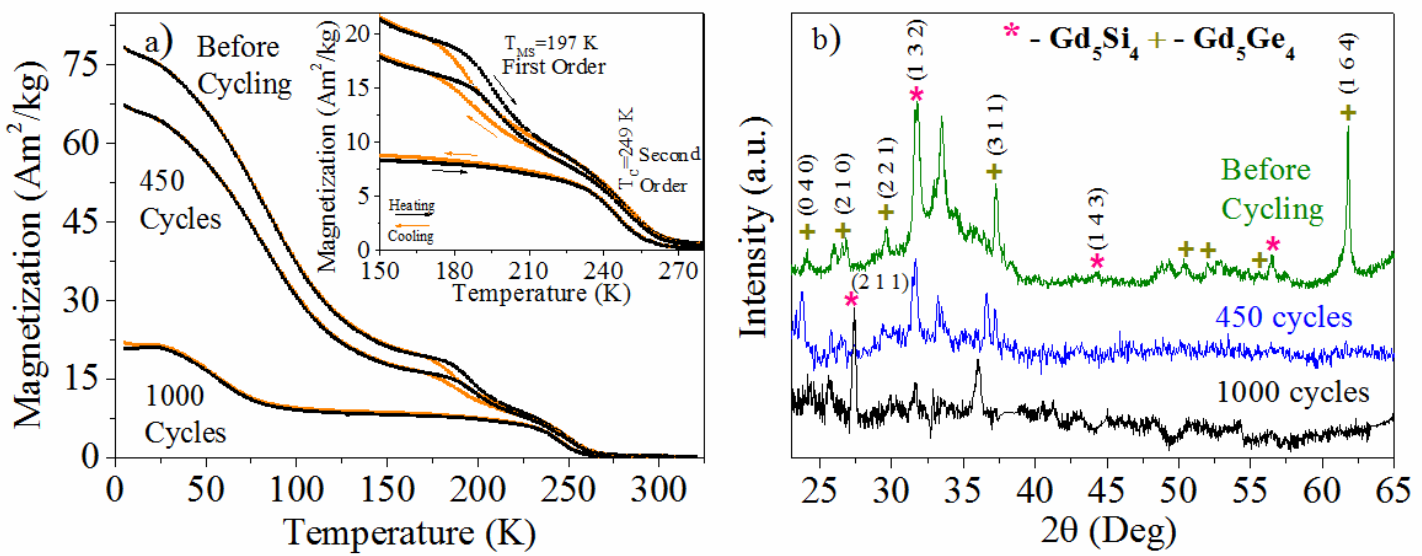

Figure 2 - (color online) a) Magnetization as a function of temperature for the $\mathrm{Gd}_{5} \mathrm{Si}_{1.3} \mathrm{Ge}_{2.7}$ thin film: before cycling, after 450 and 1000 thermal cycles in cooling and heating with a field of 0.1T; Inset: magnification between [150;280] $\mathrm{K}$; b) X-ray diffractograms of the samples: before cycling (green), after 450 cycles (blue) and after 1000 cycles (black). The peaks marked with [*] correspond to the $\mathrm{O}(\mathrm{I}) \mathrm{Gd}_{5} \mathrm{Si}_{4}$-like structure and the ones marked with [+] correspond to $\mathrm{O}$ (II) $\mathrm{Gd}_{5} \mathrm{Ge}_{4}$-like structure. 


\section{Figure 3}
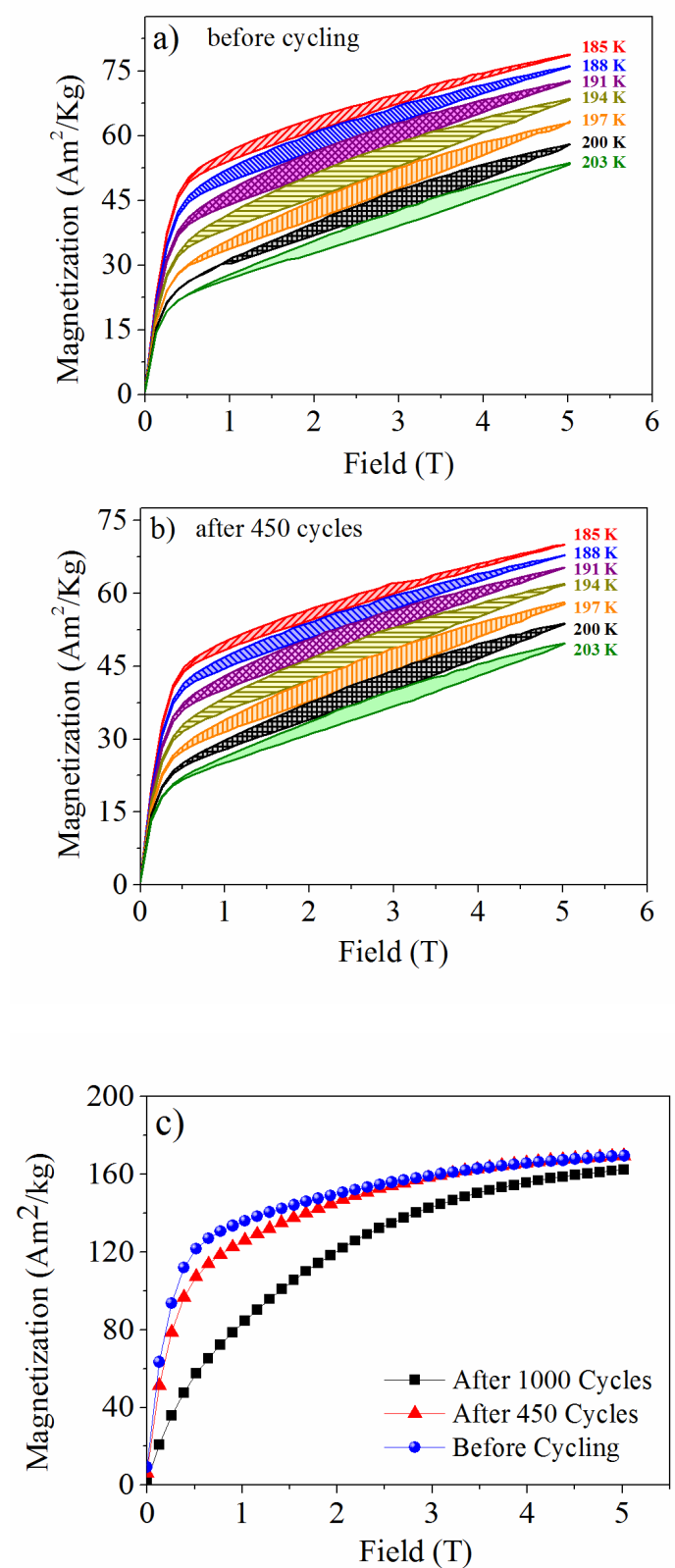

Figure 3 - (color online) Magnetization versus Field ( $\mathrm{MvsH})$ curves at temperatures around the first order transition for the samples: a) before cycling and b) after 450: and c) MvsH at $5 \mathrm{~K}$ for the samples: before cycling, after 450 and 1000 cycles. 


\section{Figure 4}
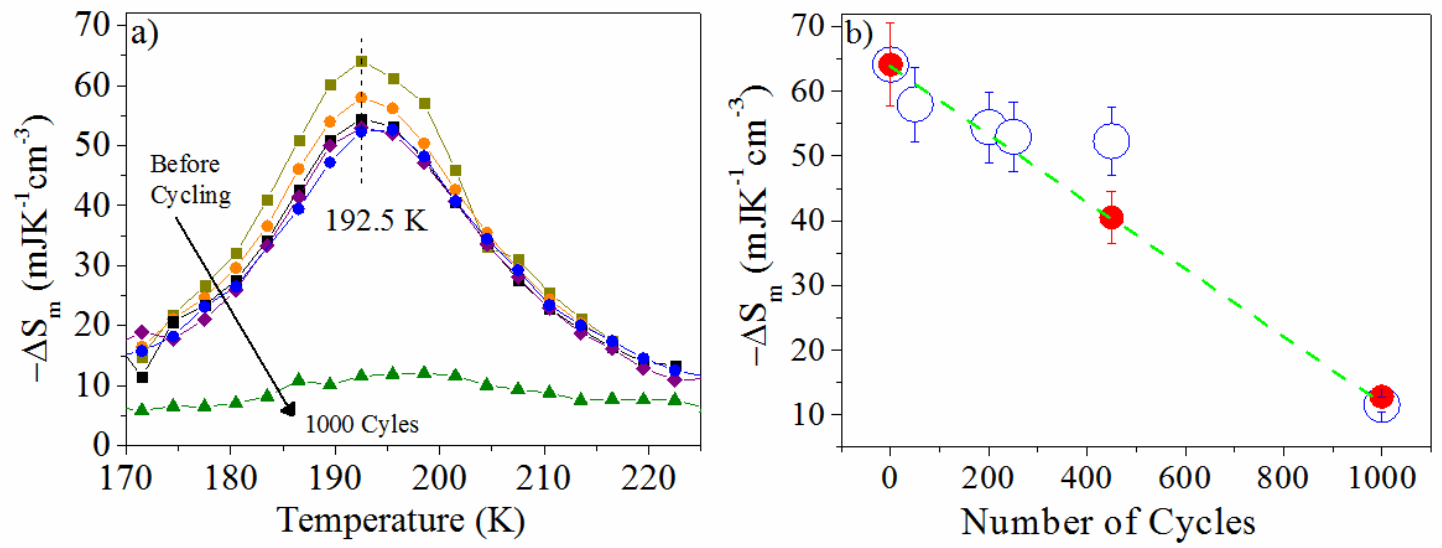

Figure 4 - (color online) a) Magnetic entropy change in the [171-225] K temperature range of the $\mathrm{Gd}_{5} \mathrm{Si}_{1.3} \mathrm{Ge}_{2.7}$ thin film before cycling, after 50, 200, 250, 450, and 1000 cycles under an applied magnetic field change of $\Delta \mathrm{H}=5 \mathrm{~T}$ b) Representation of the magnetic entropy change peak value as a function of the number of cycles for the two sequences of samples: Sample 1 (hollow circles) and Sample 2 (solid circles). 